\title{
Eugeniamyia dispar EM PITANGUEIRA: PARASITOIDES ASSOCIADOS, DINÂMICA POPULACIONAL E DISTRIBUIÇÃO DE GALHAS NA PLANTA ${ }^{1}$
}

\author{
ADRIANA NEUTZLING BIERHALS², DORI EDSON NAVA³ ${ }^{3}$ VALMIR ANTONIO COSTA², \\ VALÉRIA CID MAIA ${ }^{4}$, GABRIELA INÉS DIEZ-RODRÍGUEZ ${ }^{2}$
}

RESUMO - A pitangueira Eugenia uniflora L. (Myrtaceae) é uma frutífera nativa da América do Sul, podendo ser encontrada em quase todo o território brasileiro, bem como no Paraguai, no Uruguai e na Argentina. Nas folhas dessa planta, são encontradas galhas induzidas por Eugeniamyia dispar (Diptera: Cecidomyiidae). O objetivo deste trabalho foi determinar os parasitoides associados a $E$. dispar, a dinâmica populacional de $E$. dispar e dos parasitoides e a distribuição de galhas na planta. Ramos e folhas foram coletados quinzenalmente, durante as safras agrícolas de 2007/2008 e 2008/2009, em pomares de pitangueira localizados no município de Pelotas-RS, e transportados para o laboratório para a contagem do número de galhas. Do material coletado, foram individualizadas dez folhas em recipientes plásticos $(200 \mathrm{~mL})$ contendo $30 \mathrm{~mL}$ de uma solução à base de ágar-água ( $2 \%$ ) e metilpara-hidroxibenzoato (nipagin) ( $0,2 \%)$. Os recipientes foram fechados e mantidos sob condições controladas de temperatura $\left(24 \pm 2^{\circ} \mathrm{C}\right)$, umidade relativa $(70 \pm 10 \%)$ e fotofase $(14 \mathrm{~h})$, sendo registrado, diariamente, o número de insetos emergidos. Obteve-se uma espécie de Hymenoptera, Rileya hegeli (Eurytomidae), como parasitoide de E. dispar. Quanto à dinâmica populacional de E. dispar e de $R$. hegeli, foi observado, nos dois anos de avaliação, que a população de ambos aumentou a partir de outubro, atingindo as maiores populações nos meses de dezembro, janeiro e fevereiro, decrescendo posteriormente. O número de galhas por folha variou de zero a 23 , sendo que $61,19 \%$ das folhas apresentavam de uma a oito galhas e que o número de folhas com galhas por ramo variou de uma a nove, sendo que $63,00 \%$ dos ramos apresentaram de uma a três folhas infestadas.

Termos para indexação: Eugenia uniflora, insetos galhadores, parasitoides, inseto-praga.

\section{Eugeniamyia dispar IN SURINAM CHERRY: ASSOCIATED PARASITOIDS, POPULATION DYNAMICS AND DISTRIBUTION OF PLANT GALLS}

\begin{abstract}
The Surinam cherry, Eugenia uniflora L. (Myrtaceae), is a native fruit tree of South America and it can be found throughout the Brazilian territory, as well as in Paraguay, Uruguay and Argentina. Galls induced by Eugeniamyia dispar (E. dispar) are found on the leaves of these trees. This study aims at determining the parasitoids associated to the $E$. dispar, the population dynamic of both $E$. dispar and parasitoids and the plant gall distribution. Branches and leaves were collected every two weeks during the 2007/08 and 2008/09 crops in Surinam cherry orchards situated in the city of Pelotas, state of Rio Grande do Sul, and taken to the laboratory for gall counting. Out of the collected material, ten leaves were isolated per sample into plastic containers $(200 \mathrm{ml})$ with $30 \mathrm{ml}$ of agar solution $(2 \%)$ and methylparahydroxybenzoate (nipagin) $(0.2 \%)$. The containers were closed and kept under temperature-controlled conditions $\left(24 \pm 2^{\circ} \mathrm{C}\right)$, relative humidity $(70 \pm 10 \%)$ and photophase (14h). The number of emerged insects was daily reported. As a result, one species of Hymenoptera, Rileya hegeli (Eurytomidae) as a parasitoid of E. dispar was obtained. In relation to the population dynamics of the $E$. dispar and of the $R$. hegeli, during two years of evaluation it was noticed that the population of both species had an increase from October, and the largest populations were achieved in December, January and February, and then, decreasing onward. The number of galls per leave varied from zero to 23 and $61.19 \%$ of the leaves had one to eight galls. The number of leaves with galls in a branch varied from one to nine, and $63.00 \%$ of the branches had one to three infested leaves.
\end{abstract}

Index terms: Eugenia uniflora, galling insects, parasitoids, pest insect.

\footnotetext{
1(Trabalho 097-11). Recebido em: 23-03-2011. Aceito para publicação em: 26-01-2012.

${ }^{2}$ Embrapa Clima Temperado, Laboratório de Entomologia, Caixa Postal 403, 96001-970. Pelotas-RS. E-mail: drih.bier@gmail.com; nava.dori@cpact.embrapa.br; idiez@gmail.com

${ }^{3}$ Instituto Biológico, Agência Paulista de Tecnologia dos Agronegócios, Secretaria de Agricultura e Abastecimento. Caixa Postal 70 , 13012-970, Campinas-SP. E-mail: valmir@biologico.sp.gov.br

${ }^{4}$ Museu Nacional, Universidade Federal do Rio de Janeiro, Departamento de Entomologia, 20940-040, Rio de Janeiro-RJ. E-mail: maiavcid@acd.ufrj.br
} 


\section{INTRODUÇÃO}

A pitangueira Eugenia uniflora L. (Myrtaceae) é uma frutífera nativa da América do Sul, podendo ser encontrada em quase todo o território brasileiro, assim como no Paraguai, Uruguai e na Argentina (DONADIO et al., 2002). No Brasil, seu cultivo comercial vem sendo realizado nos Estados de Pernambuco e Rio Grande do Sul, com perspectivas de aumento, devido ao sabor exótico dos frutos e à riqueza em cálcio, fósforo, antocianinas, flavonoides, carotenoides e vitamina $\mathrm{C}$, indicando seu elevado poder antioxidante (SILVA, 2006). As frutas são utilizadas para o consumo in natura ou para o preparo de doces, geleias, sorvetes, sucos e infusões.

Apesar de ser uma planta rústica e de fácil cultivo, a falta de variedades comerciais de pitangueira dificulta sua produção com padrão de qualidade definido. Outro fator limitante para o seu cultivo são os problemas fitossanitários, como a ocorrência da mosca-das-frutas sul-americana Anastrepha fraterculus (WIEDEMANN, 1830) (Diptera: Tephritidae) que encontra neste hospedeiro condição favorável à sua multiplicação (SALLES, 1995). Além desta, o galhador Eugeniamyia dispar Maia, Mendonça e Romanowski 1996 (Diptera: Cecidomyiidae) tem causado danos nas folhas novas em plantas de pomares recém-implantados, assim como em mudas nos matrizeiros. Diferentemente do controle de $A$. fraterculus, realizado com relativo sucesso por meio do monitoramento com proteína hidrolisada e pela aplicação de inseticidas na forma de isca tóxica ou por cobertura, o manejo de $E$. dispar tem sido dificultado por falta de opções tanto para o monitoramento como para o controle.

Eugenia dispar realiza a postura nas folhas novas da pitangueira, e as larvas recém-eclodidas penetram no tecido foliar, induzindo à formação da galha. Segundo Mendonça e Romanowski (2002a), a galha é o resultado da hipertrofia dos tecidos da planta, causados por uma única larva. Além das galhas, ocorre a perda de clorofila das folhas, diminuindo a área fotossintética da planta. No último instar, as larvas saem da galha e pupam no solo, de onde os adultos emergem, porém vivem poucos dias nesse estágio.

No Brasil, poucos trabalhos sobre a dinâmica populacional de espécies galhadoras e de parasitoides associados foram desenvolvidos, de forma que dados sobre flutuação populacional, número de gerações anuais e flutuação nas taxas de parasitismo são escassos.

A fauna brasileira de Cecidomyiidae galhadores inclui cerca de 160 espécies (MAIA, 2005). Deste montante, apenas nove foram estudadas com esse enfoque, sendo que, para sete espécies (Bruggmania acaudata Maia, 2004; B. elongata Maia e Couri, 1992; B. robusta Maia e Couri, 1992; Clusiamyia nitida Maia, 1996; Cordiamyia globosa Maia, 1996; Dasineura globosa Maia, 1995, e Stephomyia rotundifoliorum Maia, 1993), há dados sobre a dinâmica populacional do galhador e de seus respectivos parasitoides. Para Dasineura gigantea Ângelo e Maia, os dados referem-se exclusivamente ao galhador; e para $E$. dispar, as informações restringem-se aos parasitoides (ÂNGELO, 2008; MAIA, 1999; MAIA; MONTEIRO, 1999; MAIA; TAVARES, 2000; MENDONÇA; ROMANOWSKY, 2002b).

A ação de predadores e parasitoides contribui para a manutenção do equilíbrio populacional de $E$. dispar, sendo, portanto, considerados importantes agentes bióticos de mortalidade. Dentre os inimigos naturais, Mendonça e Romanowski (2002b) registraram a ocorrência da formiga predadora Pseudomyrmex sp. (Hymenoptera: Formicidae), do parasitoide Rileya sp. (Eurytomidae) e de um micro-himenóptero (Chalcidoidea), ainda não identificado.

Para que medidas de controle sejam estabelecidas, um dos primeiros estudos a ser realizado é a determinação da época de ocorrência das pragas nos pomares, sua distribuição e seus inimigos naturais (PARRA, 2000). Desta forma, o objetivo deste trabalho foi determinar os parasitoides associados a $E$. dispar, estudar sua dinâmica populacional e de seus parasitoides, e a distribuição das galhas nas plantas de pitangueira.

\section{MATERIAL E MÉTODOS}

O estudo foi conduzido no Laboratório de Entomologia, a partir da coleta de amostras em pomar de pitangueira de aproximadamente um hectare, pertencente ao Banco Ativo de Germoplasma de Frutíferas Nativas da Região Sul do Brasil, localizado na área experimental da Embrapa Clima Temperado de Pelotas-RS, durante as safras agrícolas de 2007/2008 e 2008/2009.

Para a coleta das amostras, foram retirados cinco ramos de dez plantas de pitangueira com quatro anos de idade, de forma aleatória, a cada 15 dias. Os ramos foram coletados na parte superior das plantas, e as amostras foram acondicionadas em sacos plásticos devidamente etiquetados, os quais foram transportados ao laboratório. Uma parte do material coletado foi utilizada para a contagem do número de galhas contendo larvas de cecidomídeos. Outra parte foi utilizada para determinar a porcentagem de parasitismo, sendo os parasitoides enviados 
para identificação. Nesse caso, foram colocadas dez folhas individualizadas em recipientes plásticos de $200 \mathrm{~mL}(8,6 \times 7,1 \mathrm{~cm})$, contendo no fundo aproximadamente $30 \mathrm{~mL}$ de uma solução aquosa de ágar $(2 \%)$ e metilpara-hidroxibenzoato $(0,2 \%)$, para a conservação da folha. Os recipientes foram tampados com papel filme e mantidos em sala climatizada com temperatura de $24 \pm 2^{\circ} \mathrm{C}$, umidade relativa de $70 \pm 10 \%$ e fotofase de $14 \mathrm{~h}$. Diariamente, os recipientes foram observados para o registro da emergência de $E$. dispar ou de parasitoides, os quais foram conservados em álcool a 70\% e enviados, posteriormente, para identificação. Os cecidomídeos (adultos e imaturos) foram encaminhados ao Museu Nacional do Rio de Janeiro-RJ, onde foram montados em lâminas permanentes de microscopia óptica para a identificação do gênero, com base nas chaves de Gagné (1994) e da espécie, a partir das descrições das informações sobre a planta hospedeira e tipo de galha. Os parasitoides foram identificados com base em Gates (2008), sendo depositados na Coleção de Insetos Entomófagos "Oscar Monte", localizada no Centro Experimental do Instituto Biológico, em Campinas-SP.

A partir da contagem de galhas com larvas e pupas de E. dispar por folha, e do número de parasitoides emergidos, foi determinada a flutuação populacional de E. dispar e do parasitoide ao longo das duas safras agrícolas.

Para determinar a distribuição de E. dispar na planta, foi realizada, em laboratório, a contagem de galhas por folha, das cinco primeiras folhas do ápice dos ramos, e o número de folhas com galhas, por ramo, nas dez plantas amostradas, durante as quatro avaliações realizadas nos meses de dezembro e janeiro das duas safras agrícolas. Com os dados obtidos referentes à distribuição das galhas na planta, foi realizada uma classificação, considerando o intervalo de variação dos dados e levando-se em consideração o desvio-padrão e a média, estabelecendo-se quatro classes. Para o número de galhas por folha, as classes foram constituídas da seguinte forma: classe 1 - folhas sem infestação; classe 2 - folhas contendo de uma a oito galhas; classe 3 - folhas contendo de nove a 16 galhas, e classe 4 - folhas contendo mais de 16 galhas. Para determinar o número de folhas com galhas por ramo, foram definidas as seguintes classes: classe 1 - ramos sem folhas infestadas; classe 2 - ramos contendo de uma a três folhas com galhas; classe 3 - ramos contendo de quatro a seis folhas com galhas; classe 4 - ramos contendo mais de seis folhas com galhas.

\section{RESULTADOS E DISCUSSÃO}

\section{Ocorrência de parasitoides de $E$. dispar}

Todos os espécimes de parasitoides coletados pertenceram a uma única espécie, Rileya hegeli (Hymenoptera: Eurytomidae). Provavelmente, esta deve ser a espécie registrada também por Mendonça e Romanowski (2002b), uma vez que os autores mencionam a ocorrência de Rileya sp., em galhas de $E$. dispar nas folhas de pitangueira. Esses autores também relataram outro parasitoide micro-himenóptero (Chalcidoidea), cuja espécie não foi determinada.

Além de E. dispar, $R$. hegeli também parasita cecidomídeos não identificados em Coccoloba diversifolia Jaqc. (Polygonaceae), nas nervuras de folhas de Piper sp. (Piperaceae) e nas flores de Leucaena pulverulenta (Schlect.) (Fabaceae). O parasitoide $R$. hegeli ocorre desde o sul dos Estados Unidos da América até a América do Sul, havendo registros para Minas Gerais (Viçosa) e Rio Grande do Sul (Porto Alegre), além de Costa Rica, Estados Unidos da América, Guatemala, Jamaica, México, República Dominicana e Venezuela (GATES, 2008).

Rileya hegeli é um ectoparasitoide solitário, cujos ovos são transparentes e delgados. As fêmeas adultas perfuram as galhas com o ovipositor e colocam um ovo sobre as larvas de E. dispar do último instar larval. Após a eclosão, a larva de $R$. hegeli fixa-se ao hospedeiro e inicia a alimentação. No último instar, o parasitoide posiciona-se na galha com a cabeça voltada para a galeria e, com o desenvolvimento da pupa, esta adquire uma pigmentação de cor laranja. No momento da emergência, o parasitoide abre com suas mandíbulas um pequeno orifício oval na epiderme da galha e abandona-a (MENDONÇA, 1996).

Das galhas coletadas em campo, nos meses de dezembro e janeiro, foi observada uma taxa de parasitismo por $R$. hegeli de até $85 \%$. Mendonça e Romanowski (2002b) relatam uma taxa de 50\% por Rileya sp. Esse alto percentual de parasitismo atingido por $R$. hegeli indica que essa espécie é um importante agente de mortalidade de E. dispar em pomares de pitangueira, e que, juntamente com outras medidas de controle, pode ser incluída no manejo integrado de $E$. dispar. Além disso, $R$. hegeli poderia ser utilizado em um programa de controle biológico aplicado, havendo, porém, necessidade de estudos sobre técnicas para a sua criação e a de seu hospedeiro em laboratório. 
Dinâmica populacional de $E$. dispar e de seu parasitoide Rileya hegeli

A incidência de galhas em pitangueiras causadas por E. dispar nos dois anos de avaliação apresentou um incremento gradativo a partir do mês de outubro, no início da primavera, atingindo picos populacionais nos meses de dezembro, janeiro e fevereiro. A partir de março, o número de galhas diminuiu gradativamente até agosto, quando a incidência de galhas foi próxima de zero (Fig. 1).

A baixa quantidade de galhas com larvas ou pupas nas folhas de pitangueira durante o inverno seria decorrente das baixas temperaturas, normalmente registradas nessa época do ano, que estariam atuando sobre o desenvolvimento de E. dispar e sobre o crescimento das plantas, visto que essas praticamente não emitem brotações (FRAZON; RASEIRA, 2004).

Quanto ao nível populacional de $R$. hegeli, foram observados valores próximos de zero nos meses de julho a outubro, nos dois anos de avaliação (Fig. 1). A população cresceu gradativamente a partir do aumento da população de $E$. dispar, sendo registrados os primeiros parasitoides em novembro, e o pico populacional, em fevereiro, quando a população voltou a diminuir, paralelamente à redução da população de E. dispar (Fig. 1).

Em fevereiro, a população do parasitoide ultrapassou a da praga e, nos meses seguintes, ambas as populações foram menores, caracterizando o mecanismo da densidade recíproca (HUFFAKER; MESSENGER, 1976). Os níveis populacionais do parasitoide foram mais elevados na safra de 2008/2009 que na safra anterior, possivelmente devido à ocorrência de um período hibernal com temperaturas mais elevadas.

\section{Distribuição de galhas de $\boldsymbol{E}$. dispar em pitangueira: número de galhas por folha e número de folhas com galhas por ramo}

O número de galhas com larvas e pupas de E. dispar por folha variou de zero a 23. De todas as folhas avaliadas, $31,3 \%$ não apresentaram galhas, correspondendo à classe 1 . A maior quantidade de folhas com galhas $(61,2 \%)$ correspondeu à classe 2 , sendo seguida pela classe 3 com $6,7 \%$ das folhas infestadas, e pela classe 4 , onde apenas $0,5 \%$ das folhas apresentou mais de 16 galhas (Fig. 2A). Segundo Maia et al. (1996), o número de galhas de E. dispar por folha pode variar de uma até 30 , sendo, portanto, próxima da relatada neste trabalho. Mendonça e Romanoski (2002a) mencionam que podem ser encontradas até 33 galhas por folha e até 71 galhas por brotação. No caso de folhas que possuíam de nove a 16 galhas (classe 3), foi possível observar que as mesmas apresentavam deformações, adquirindo coloração amarela e, com a saída das larvas de E. dispar para o solo, ocorria uma necrose e o abortamento. Quando as galhas eram formadas nas folhas novas das brotações, o crescimento era paralisado, e o ponteiro dos ramos secava.

A incidência de plantas com ramos contendo folhas com galhas nos pomares de pitangueira, durante a época com maior população de $E$. dispar, nos meses de dezembro e janeiro, foi variável, pois em $22 \%$ (classe 1) dos ramos avaliados não foram observadas folhas com galhas e apenas $4 \%$ dos ramos (classe 4) apresentaram mais de seis folhas infestadas (Fig. 2B). A maior porcentagem de folhas infestadas $(63 \%)$ nos ramos ocorreu quando foram observadas de uma a três folhas com galhas (classe 2), seguida de classe 3 , com $11 \%$, apresentando de quatro a seis folhas com galhas.

Normalmente, as folhas infestadas foram as mais jovens e mais próximas do ápice, sendo que após o ataque eram abortadas. Assim, em plantas atacadas por E. dispar, é comum observar ramos do ano sem folhas.

Embora E. dispar seja considerada uma das principais pragas do cultivo de pitangueira, as plantas mais atacadas são as recém-implantadas, em pomares de até quatro anos. Entretanto, são as plantas dos viveiros as mais prejudicadas, pois neste ambiente os fatores abióticos, como chuva e temperatura, e os bióticos, como o parasitismo e a predação não ocorrem com a mesma intensidade que em condições naturais. 


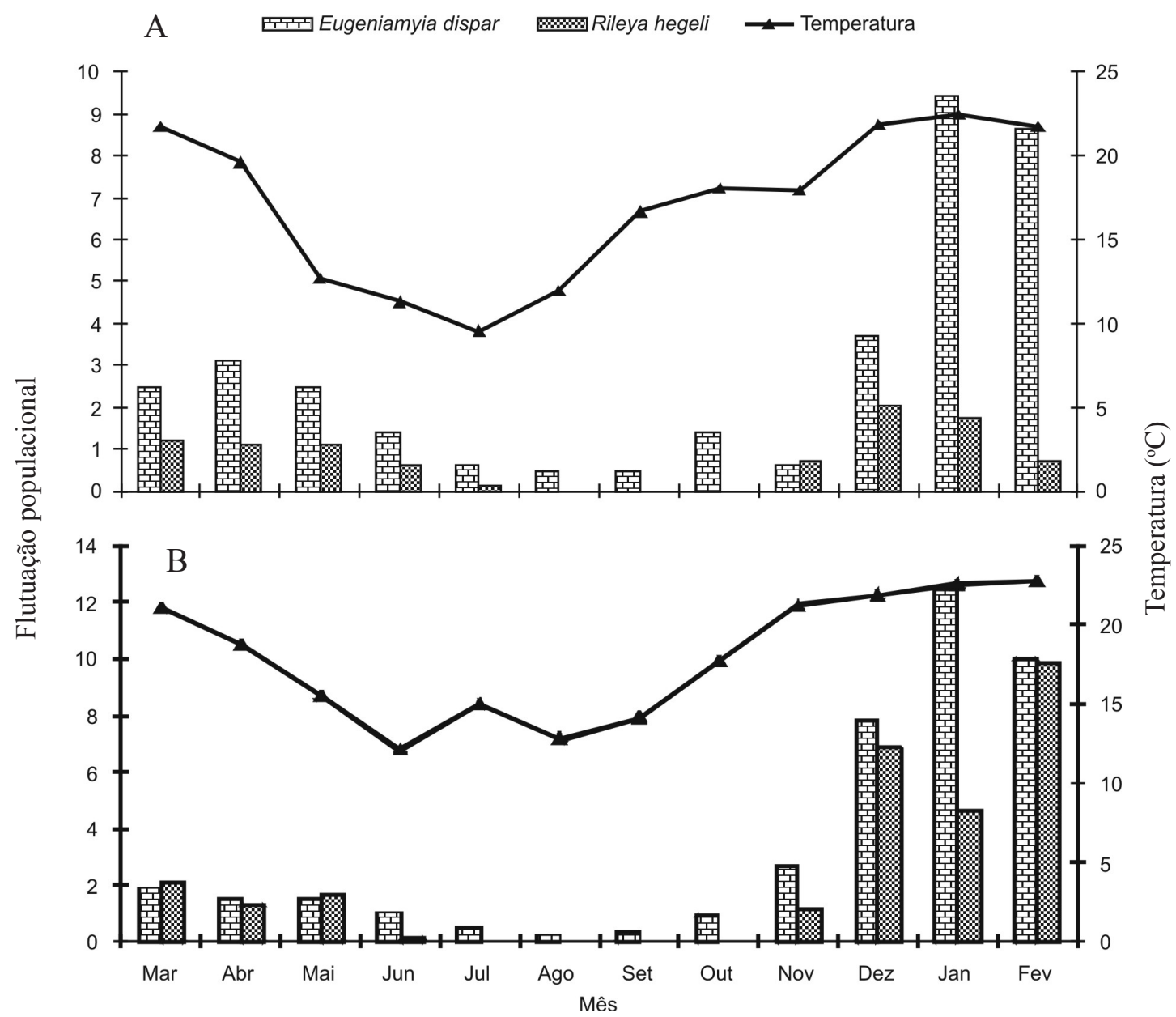

FIGURA 1 - Flutuação populacional do galhador Eugeniamyia dispar e do seu parasitoide Rileya hegeli em pitangueira (Eugenia uniflora). A) Safra agrícola de 2007/2008 e B) Safra agrícola de 2008/2009. Embrapa Clima Temperado. Pelotas-RS. 


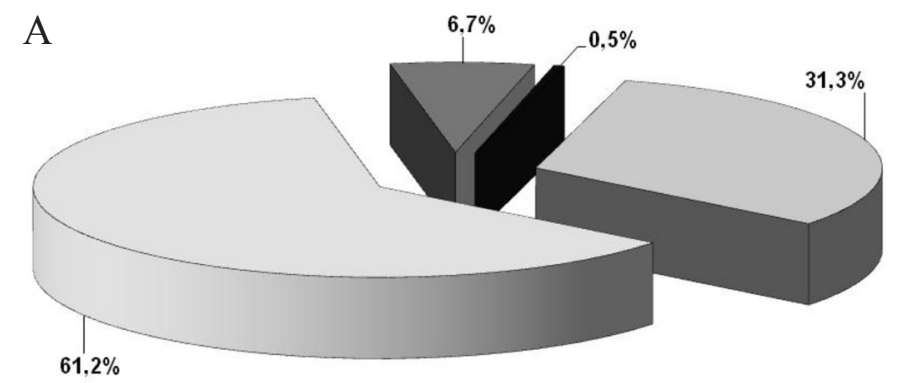

$\square$ Classe $1(0)$

口Classe $2(1-8)$

$\square$ Classe $3(9-16)$

- Classe $4(>17)$

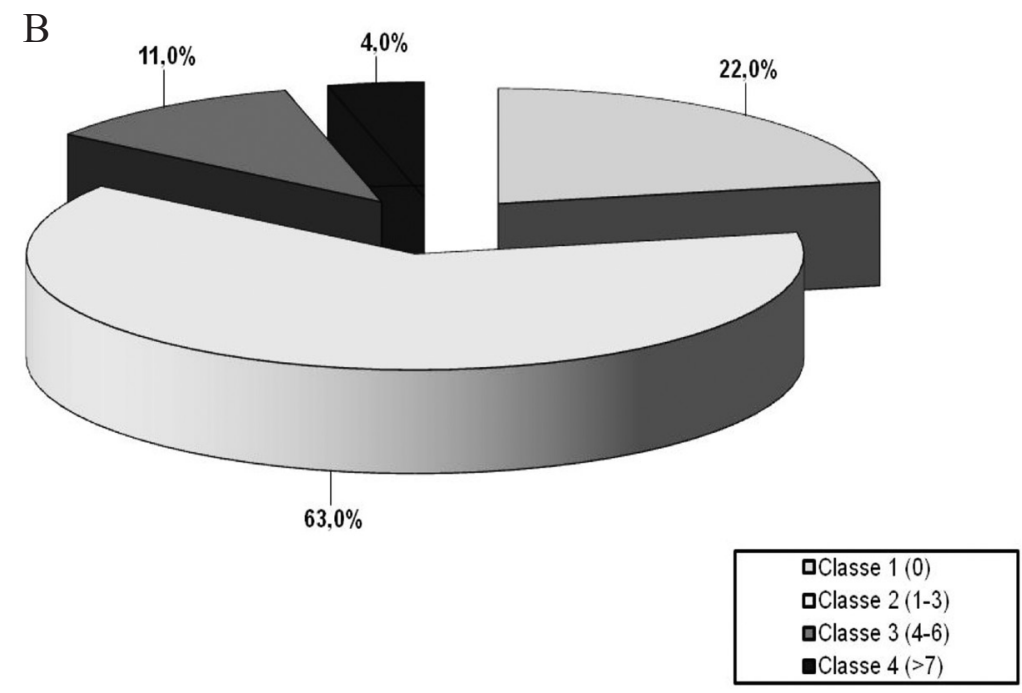

FIGURA 2 - Distribuição de galhas de Eugeniamyia dispar em pitangueira (Eugenia uniflora). A) Número de galhas por folha; B) Número de folhas com galhas por ramo. Embrapa Clima Temperado, Pelotas-RS.

\section{CONCLUSÃO}

1-Rileya hegeli é um parasitoide de Eugeniamyia dispar, praga da pitangueira cultivada em pomar no município de Pelotas.

2-Dezembro, janeiro e fevereiro são os meses de maior ocorrência de E. dispar e de R. hegeli.

\section{AGRADECIMENTOS}

Ao Engenheiro Agrônomo MSc.Odimar Zanuzo Zanardi, pelo auxílio na elaboração dos gráficos.

\section{REFERÊNCIAS}

ÂNGELO, A.C. Ciclo de vida de Dasineura gigantea Angelo \& Maia, 1999 (Diptera, Cecidomyiidae). Floresta, Curitiba, v.38, n.1, p.23-32, 2008.

DONADIO, L.C.; MÔRO, F.V.; SERVIDONE, A.A. Frutas brasileiras. Jaboticabal: Novos Talentos, 2002. 288p

FRAZON, R.C.; RASEIRA, M.C.B. Características fenológicas e morfológicas, floração e maturação dos frutos de mirtáceas frutíferas nativas do Sul do Brasil. In: RASEIRA, M.C.B.; ANTUNES, L.E.C.; TREVISAN, R.; GONÇALVES, E.D. Espécies frutíferas nativas do Sul do Brasil. Pelotas: Embrapa Clima Temperado, 2004. p. 27-46. 
GAGNÉ, T.J. The gall midges of the neotropical region. Ithaca: Cornell University Press, 1994. 356 p.

GATES, M.W. Species revision and generic systematics of world Rileyinae (Hymenoptera: Eurytomidae). Berkeley: University of California Press, 2008. 332p.

HUFFAKER, M.; MESSENGER, P.S. Theory and practice of biological control. New Yiork:Academic Press, 1976. 788p.

MAIA, V.C. Artrópodes associados às galhas de Cecidomyiidae (Diptera) em Eugenia rotundifolia (Myrtaceae) e Clusia lanceolata (Clusiaceae) em uma restinga do Estado do Rio de Janeiro, Brasil. Iheringia, Série Zoologia, Porto Alegre, v.87, p.7579, 1999.

MAIA, V.C. Catálogo dos Cecidomyiidae (Diptera) do Estado do Rio de Janeiro. Biota Neotropica, São Paulo, v.5, n.2, p.1-15, 2005.

MAIA, V.C.; MENDONÇA, M.S.; ROMANOWSKI, H.P. Eugeniamyia dispar (Diptera, Cecidomyiidade, Lasiopteridi) associated with Eugenia uniflora L. (Myrtaceae) in Brazil. Revista Brasileira de Zoologia, Curitiba, v. 13, p. 1087-1090, 1996.

MAIA, V.C.; MONTEIRO, R.F. Espécies cecidógenas (Diptera, Cecidomyiidae) e parasitoides (Hymenoptera) associados a Guapira opposita (Vell.) Reitz (Nyctaginaceae) na restinga da Barra de Maricá, Rio de Janeiro. Revista Brasileira de Zoologia, Curitiba, v.16, n.2, p.483-487, 1999.
MAIA, V.C.; TAVARES, M.T. Cordiamyia globosa Maia, 1996 (Diptera, Cecidomyiidae) - flutuação populacional e parasitoides associados. Revista Brasileira de Zoologia, Curitiba, v.17, n.3, p.589593, 2000.

MENDONÇA, M.S. Ecologia de Eugeniamyia dispar (Diptera: Cecidomyiidae), galhador sobre Eugenia uniflora (Myrtaceae). 1996. Dissertação (Mestrado) - Instituto de Biociências, Universidade Federal do Rio Grande do Sul, Porto Alegre, 1996.

MENDONÇA, M.S.; ROMANOWSKI, H.O. Life history on the gall-maker Eugeniamyia dispar Maia, Mendonça e Romanowski 1996 (Diptera, Cecidomyiidae). Brazilian Journal of Biology, São Carlos, v.62, n.2, p.277-283, 2002a.

MENDONÇA, M.S.; ROMANOWSKI, H.O. Natural enemies of the gall-maker Eugeniamyia dispar (Diptera, Cecidomyiidae). Brazilian Journal of Biology, São Carlos, v.62, n.2, p.269-275, 2002 b.

PARRA, J.R.P. A biologia de insetos e o manejo de pragas: da criação em laboratório à aplicação em campo. In: GUEDES, J.C.; COSTA, I.D.; CASTIGLIONI, E. Bases e técnicas do manejo de insetos. Santa Maria: UFSM/CCR/DFS, 2000. p. 1-29.

SALLES, L.A.B. Bioecologia e controle da mosca-das-frutas sul-americana. Pelotas: Embrapa CPACT, 1995. 58p.

SILVA, S.M. Pitanga. Revista Brasileira de Fruticultura, Jaboticabal, v. 28, n. 1, p. 1-1, 2006. 